\title{
The role of targeted regulation of COX11 by miR-10a-3p in the development and progression of paediatric mycoplasma pneumoniae pneumonia
}

\author{
Wenhong $\mathrm{Li}^{1}$, Xin Ding ${ }^{1}$, Rui Zhao ${ }^{2}$, Donghui Xiong ${ }^{3}$, Zhiping Xie ${ }^{4}$, Jing Xu' ${ }^{1}$, Meiling Tan ${ }^{1}$ Chunyu $\mathrm{Li}^{5}$, \\ Chunfu Yang ${ }^{1}$
}

${ }^{1}$ Department of Paediatrics, The First Affiliated Hospital of the Qiqihar Medical University, Qiqihar, China; ${ }^{2}$ Department of Medical Records, The First Affiliated Hospital of the Qiqihar Medical University, Qiqihar, China; ${ }^{3}$ Department of Personnel, The First Affiliated Hospital of the Qiqihar Medical University, Qiqihar, China; ${ }^{4}$ Department of Epidemiology and Health Statistics Public Health School of Qiqihar Medical University, Qiqihar, China; ${ }^{5}$ Department of Paediatrics, The First Affiliated Hospital of Jiamusi University, Jiamusi, China

Contributions: (I) Conception and design: W Li, C Yang; (II) Administrative support: D Xiong; (III) Provision of study materials or patients: X Ding, J Xu, C Li; (IV) Collection and assembly of data: R Zhao, M Tan, Z Xie; (V) Data analysis and interpretation: C Yang, W Li, Z Xie; (VI) Manuscript writing: All authors; (VII) Final approval of manuscript: All authors.

Correspondence to: Chunfu Yang. Department of Paediatrics, The First Affiliated Hospital of the Qiqihar Medical University, 26 Xiangyang Street, Fularji District, Qiqihar, China. Email: yangyangyang_1700@163.com.

Background: MiR-10a-3p is associated with the pathogenesis of many immune inflammatory diseases including Mycoplasma pneumoniae pneumonia (MPP), and cytochrome coxidase assembly homologue 11 (COX11) is one of its direct target proteins. This study investigates the function and mechanism of miR-10a3 p targeting with COX11 in the development and progression of paediatric MPP.

Methods: Ninty-seven paediatric MPP patients and 100 age- and sex-matched healthy children were enrolled. Clinical and laboratory indicators of paediatric MPP patients were collected. The mRNA levels of the COX11 gene and miR-10a-3p were detected by qRT-PCR. THP-1 mononuclear macrophages were stimulated using MPP lipid-associated membrane proteins (Mp-LAMPs). The relative expression level of miR-10a-3p was detected after 12, 24, and 48 h. THP-1 cells were transfected to overexpress or inhibit the expression of miR-10a-3p, miR-10a-3p, COX11 mRNA, NF- $\kappa$ B signalling pathway-related proteins, and C-reactive protein (CRP) were detected after $48 \mathrm{~h}$ by Western blot.

Results: The relative expression level of miR-10a-3p in the MPP group was $2.38 \pm 0.52$, compared with $1.76 \pm 0.38$ in control group $(\mathrm{t}=4.584, \mathrm{P}<0.001)$ while $C O X 11$ in MPP group was $3.70 \pm 1.12$, compared to $5.78 \pm 1.84$ in control group $(\mathrm{t}=4.876, \mathrm{P}<0.001)$. Pearson correlation analysis showed that miR-10a-3p and COX11 in MPP group presented a negative correlation $(\mathrm{r}=-0.679, \mathrm{P}<0.001)$. By searching in the prediction website of TargetScan database, it was found that miR-10a-3p and Cox11 genes had targeted regulatory binding sites, and the targeting relationship between miR-10a-3p and Cox11 genes was confirmed by dual luciferase reporting assay in $293 \mathrm{~T}$ cells. Among paediatric MPP patients, miR-10a-3p expression had a positive correlation with the white blood cells count, erythrocyte sedimentation rate (ESR), and CRP expression, while COX11 mRNA expression had a positive correlation with ESR and CRP. After LAMP stimulation, the miR-10a-3p expression level in THP-1 cells significantly increased $(\mathrm{P}<0.05)$. After THP1 cells were transfected with the miR-10a-3p mimic or inhibitor, the relative expression level of miR-10a$3 \mathrm{p}$ significantly increased or decreased, respectively. COX11 expression in the mimic group significantly decreased, whereas COX11 in the inhibitor group significantly increased (both $\mathrm{P}<0.05$ ). In addition, after transfection, I $\mathrm{B} \mathrm{B} \alpha$ expression significantly decreased and that of $\mathrm{p}-\mathrm{IKK} \alpha / \beta, \mathrm{p}-\mathrm{p} 65$, and CRP significantly increased in the mimic group, and the opposite was true in the inhibitor group.

Conclusions: In paediatric MPP, increased miR-10a-3p downregulated COX11, activating NF- $\kappa \mathrm{B}$ signalling pathway to promote disease development and progression. 


\begin{abstract}
Keywords: miR-10a-3p; cytochrome coxidase assembly homologue 11 (COX11); Mycoplasma pneumoniae pneumonia (MPP); NF-kB signal pathway; C-reactive protein
\end{abstract}

Submitted Apr 28, 2021. Accepted for publication Jul 23, 2021.

doi: $10.21037 /$ jtd-21-710

View this article at: https://dx.doi.org/10.21037/jtd-21-710

\section{Introduction}

Mycoplasma pneumoniae pneumonia (MPP) is a common respiratory tract disease (1). Children are prone to MPP due to their weaker resistance and environmental factors (2). Epidemiological survey studies have shown that the prevalence of MPP in community-acquired pneumonia among children over 5 years old was more than $40 \%$. Severe MPP development not only causes respiratory symptoms such as asthma attacks but also can damage extrapulmonary systems, greatly impairing the health and quality of life of the child and increasing the burden on the family and society $(3,4)$. Therefore, investigation of the pathogenesis of paediatric MPP and research into effective therapeutic targets are important for its early diagnosis and treatment.

The pathogenesis of MPP is not completely clear. The immune mechanism plays an important role in Mycoplasma pneumoniae infection and MPP development and progression $(5,6)$. On one hand, Mycoplasma pneumoniae can induce humoural immune responses in the body to stimulate B cells to produce immunoglobulins to form immune complexes. On the other hand, the surface antigen of Mycoplasma pneumoniae is similar to the surface antigens of many tissues and organs of the human body, and this antigen can change the expression of some surface antigens on human cells to induce immune cells to attack self tissues, resulting in immune disorders in infected patients (7).

MicroRNAs (miRNAs) are small RNAs with a length of approximately 10-25 nucleotides that play important roles in transcription and post-transcriptional gene regulation. miRNAs are the most abundant small RNA family. They are conserved among all eukaryotes and play a global regulatory role in cell proliferation, cell differentiation, homeostasis, disease progression, and immune and inflammatory responses $(2,8)$. The main function of miRNAs is to silence or downregulate target gene expression. They have high specificity in the regulation of their target genes. In addition, due to the time and tissue specificity of their regulation, miRNAs can perform very precise regulation of many biological processes (9). The expression levels of miRNAs are altered in various diseases, including autoimmune diseases, cancer, and infectious diseases, and they are associated with the pathogenesis of the diseases $(2,8,10)$. One study $(11)$ showed that miR-10a-3p expression was significantly upregulated in respiratory syncytial virus (RSV)-related paediatric pneumonia and was associated with the mechanism of the development of RSV-related paediatric pneumonia. RSV can induce cells to produce the very destructive reactive oxygen species (ROS). They induce cells to release signals to the immune system that cause its over-reaction, producing inflammatory responses similar to that during asthma attacks, and upregulation of miR10a-3 expression promotes this inflammatory process. MPP is an inflammatory disease of the lungs caused by microbial infection. Immune and inflammatory responses also play important roles in its pathogenesis $(12,13)$. You et al. (14) showed that miR-10a-3p in lupus nephritis participated in the immune response reaction and mediated inflammatory responses by regulating the $\mathrm{T}$ helper $17 /$ regulatory $\mathrm{T}$ cell balance. Therefore, we speculated that miR-10a-3p might be associated with the pathogenesis of MPP.

Bioinformatics results have shown that cytochrome $c$ oxidase assembly homologue 11 (COX11) is a target of miR10a-3p. It participates in metabolism, apoptosis, energy supply, and reactive oxygen production in organisms and plays an important role in cancer and respiratory system diseases $(15,16)$. There is no report on the function of COX11 in MPP. Therefore, in this study, we aimed to investigate the role of targeted regulation of COX11 gene expression by miR-10a-3p in the development and progression of paediatric MPP.

We present the following article in accordance with the TREND reporting checklist (available at https://dx.doi. org/10.21037/jtd-21-710).

\section{Methods}

\section{General information}

Paediatric MPP patients who sought treatment for the first 
Table 1 Primer sequences for miR-10a-3p, COX11, and $\beta$-actin

\begin{tabular}{ll}
\hline mRNA & PCR primers \\
\hline miR-10a-3p & Forward: 5'-AATTGAAGGAGAGTAACAGCTC-3' \\
& Reverse: 5'-TCATCCTCCGCTATTTGTCATC-3' \\
cox11 & Forward: 5'-TGCGGAGTCAGTAGTCATGTTTC-3' \\
& Reverse: 5'-CGTGGCGTGTGGCATGTA-3' \\
$\beta$-actin & Forward: 5'-TACCCTTCGTATCTCTTATCGC-3' \\
& Reverse: 5'-GGTATTTCAATGGGTATGAGTG-3'
\end{tabular}

time in The First Affiliated Hospital of the Qiqihar Medical University between January 2019 and September 2020 were enrolled as the study subjects. The inclusion criteria were (I) patients aged $\leq 14$ years old; (II) patients who were diagnosed based on the diagnostic criteria of MPP in the $8^{\text {th }}$ edition of Practical Paediatrics; (III) patients who did not receive macrolides, glucocorticoids, or immunomodulators for treatment; and (IV) patients who did not have a history of other acute infectious diseases within 3 months. The exclusion criteria were (I) patients who had other respiratory system diseases, such as bronchial asthma or tuberculosis; (II) patients who had combined metabolic diseases, blood system diseases, autoimmune diseases, or other inflammatory diseases; and (III) the children or their guardians did not want to participate in this study. A total of 97 paediatric MPP patients were enrolled. Their ages ranged from 314 years. The average age was $6.31 \pm 1.21$ years old. There were 47 male patients and 50 female patients. In addition, 100 age- and sex-matched healthy children who underwent a physical examination in our hospital in the same period were enrolled as the normal control group. Their ages ranged from 4-14 years old, their average age was $6.95 \pm$ 1.52 years, and there were 50 male and 50 female children. The inclusion criteria of the control group were (I) children aged $\leq 14$ years, (II) children or their guardians who volunteered to participate in this research, and (III) children who had had no acute infectious diseases within 3 months. The exclusion criteria were: (I) children who had respiratory system diseases, (II) children who had metabolic diseases, blood system diseases, autoimmune diseases, or other inflammatory diseases, and (III) children who had heart, brain, liver, or kidney diseases. The comparison of ages and sexes between paediatric MPP patients and the control group did not show a significant significance $(\mathrm{P}>0.05)$. All procedures in this study conformed with the Declaration of Helsinki and were approved by the Ethics Committee of the
First Affiliated Hospital of the Qiqihar Medical University (number: 2020020). All study subjects or guardians signed informed consent.

\section{Study methods}

\section{Collection of clinical and laboratory indicators}

Medical records were reviewed to collect the sex, age, and clinical presentations (such as cough, fever, wheezing, nasal congestion, runny nose, and pulmonary rales) of the paediatric MPP patients. The white blood cell (WBC) count, neutrophil (Neut) count, platelet (PLT) count, erythrocyte sedimentation rate (ESR), and C-reactive protein (CRP) level were recorded.

\section{Validation of the targeting relationship between miR- 10a-3p and the COX11 mRNA}

The results of our search of the online miRNA target prediction website TargetScan showed that miR-10a-3p and the COX11 mRNA had potential binding sites. The targeting relationship between these two was validated by a dual luciferase reporter assay in 293T cells. The detection procedures were performed according to the instructions of the dual luciferase reporter experiment reagent kit (BrightGlo $^{\mathrm{TM}}$ luciferase detection system, Promega, USA, lot: E2610/E2620/E2650. Origin: USA).

\section{Detection of the relative expression levels of miR-10a- $3 p$ and COX11 mRNA}

Peripheral blood (5 mL) was collected from paediatric MPP patients and children in the healthy control group and placed in an ethylenediamine tetraacetic acid anti-coagulant tubes. Total RNA was extracted from peripheral blood using the Trizol reagent. The RNA concentration was measured in a ultra-violet (UV) spectrophotometer. RNA was reversetranscribed into cDNA using the PrimeScript ${ }^{\mathrm{TM}} \mathrm{RT}$ reagent kit (Takara Bio, Japan; lot: RR037A). The expression levels of COX11 mRNA and miR-10a-3p were detected using the SYBR Green reagent kit (Takara Bio, Japan; lot: BL698A) in a quantitative real-time polymerase chain reaction (qRTPCR) system. $\beta$-Actin was used as the internal control. Primer sequences for miR-10a-3p, COX11, and $\beta$-actin are shown in Table 1. The total reaction system had a volume of $20 \mu \mathrm{L}$. The reaction condition was pre-denaturation at $94{ }^{\circ} \mathrm{C}$ for $10 \mathrm{~min}$ followed by 40 cycles of denaturation at $95{ }^{\circ} \mathrm{C}$ for $15 \mathrm{~s}$, annealing at $60{ }^{\circ} \mathrm{C}$ for $30 \mathrm{~s}$, and extension at $75^{\circ} \mathrm{C}$ for $1 \mathrm{~min}$. After the reaction was completed, the obtained data were used for relative quantitative analysis of 
the expression of miR-10a-3p and COX11 mRNA using the standard curve method. The results were calculated using the $2^{-\Delta \Delta \mathrm{CT}}$ method.

\section{Stimulation of THP-1 cells by Mp-LAMPs}

THP-1 mononuclear macrophages were inoculated into the culture medium containing $10 \%$ foetal bovine serum (FBS). The culture conditions were $37{ }^{\circ} \mathrm{C}$ and $5 \% \mathrm{CO}_{2}$. The experiment was performed when cells were in the logarithmic growth period. THP-1 cells were inoculated into 24-well plates at $1 \mathrm{~mL} /$ well. Mp-LAMPs were used for stimulation at the concentration of $2 \mu \mathrm{g} / \mathrm{L}$. A blank control was set up, and three parallel samples were collected. The relative expression levels of miR-10a-3p in the two groups of THP-1 cells were detected by qRT-PCR at 12, 24, and $48 \mathrm{~h}$.

\section{Treatment of THP-1 cells with the miR-10a-3p mimic and inhibitor}

The miR-10a-3p mimic and inhibitor and their negative controls (mimic NC and inhibitor NC) were synthesized and provided by Shanghai GenePharma Co. Ltd. The miR-10a-3p mimic, inhibitor, mimic NC, or inhibitor NC was transfected into THP-1 cells in the logarithmic growth stage. Cells were collected after $48 \mathrm{~h}$ to detect the expression levels of miR-10a-3p and COX11 mRNA in THP-1 cells after transfection.

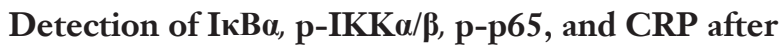 transfection}

Western blot was used to detect proteins. THP-1 cells $48 \mathrm{~h}$ after transfection were collected and washed with PBS three times. The protein lysis buffer was added and centrifuged at $14,000 \mathrm{r} / \mathrm{min}$ for $30 \mathrm{~min}$. The supernatant after centrifugation was collected, and the protein concentration was measured using the BCA reagent kit. The protein sample buffer was added, and the sample was boiled in boiling water for denaturation. Proteins were separated by sodium dodecyl sulfate-polyacrylamide gel electrophoresis at $80 \mathrm{~V}$ for $2 \mathrm{~h}$ and then transferred onto a poly(vinylidene fluoride) membrane using wet electroblotting at $350 \mathrm{~mA}$ for $2 \mathrm{~h}$. The membrane was incubated with $5 \%$ bovine serum albumin at room temperature for $1 \mathrm{~h}$, incubated with primary antibodies $[\mathrm{I} \kappa \mathrm{B}-\alpha$ rabbit monoclonal antibody (ab32518, 1:1,000, Abcam, UK), p-IKK $\alpha / \beta$ rabbit monoclonal antibody (ab92700, 1:1,000, Abcam, UK), p-p65 rabbit monoclonal antibody (ab28849, 1:1,000, Abcam, UK), CRP rabbit monoclonal antibody (ab22048,
1:1,000, Abcam, UK), glyceraldehyde-3-phosphate dehydrogenase (GAPDH) rabbit monoclonal antibody (A119056, 1:1,000, Abclonal, China)] at $4{ }^{\circ} \mathrm{C}$ overnight, then incubated with goat anti-rabbit IgG horseradish peroxidase secondary antibody (ab7090, 1:1,000, Abcam, $\mathrm{UK}$ ) at room temperature for $2 \mathrm{~h}$. GAPDH was used as the internal control. Protein bands were exposed using the electrogenerated chemiluminescence solution, developer solution, and fixing solution. The grey density values of protein bands were detected using ImageJ software. The relative expression level of the target protein was the grey density value of the target protein divided by the grey density value of GAPDH protein.

\section{Statistical analysis}

All statistical analyses were performed with SPSS22.0 software. Count data are expressed as the frequency and percentage, and the groups were compared by the chisquared test. Quantitative data that conformed to the normal distribution are described as mean \pm standard deviation $(\bar{x} \pm \mathrm{s})$, the comparison of data between two groups was performed using the test, and the pairwise comparison of data between multiple groups was performed using the Student-Newman-Keuls q-test. The correlation between two normally distributed quantitative variables was analysed by Pearson correlation. $\mathrm{P}<0.05$ indicated that the difference had statistical significance.

\section{Results}

Correlations between the relative expression levels of miR$10 a-3 p$ and COX11 mRNA and the laboratory indicators of paediatric MPP patients

The common clinical presentations and laboratory examination results of paediatric MPP patients are shown in Table 2. The major clinical symptoms, from most to least common, were cough in $93(95.9 \%)$ patients, fever in 70 $(72.2 \%)$ patients, pulmonary rales in $62(63.9 \%)$ patients, wheezing in $21(21.6 \%)$ patients, and nasal congestion and runny nose in $20(20.6 \%)$ patients.

The relative expression level of miR-10a-3p in the MPP group was $2.38 \pm 0.38$, and the relative expression level of miR-10a-3p in the healthy control group was $1.76 \pm 0.25$ $(\mathrm{t}=4.584, \mathrm{P}<0.001)$ (Figure $1 A)$. The relative expression level of COX11 mRNA in the MPP group was $3.70 \pm 0.87$, and the relative expression level of COX11 mRNA in the 
healthy control group was $5.78 \pm 1.24(\mathrm{t}=4.876, \mathrm{P}<0.001)$ (Figure $1 B$ ). Pearson correlation analysis of the expression levels of miR-10a-3p and COX11 mRNA in the MPP group showed that these two had a negative correlation $(\mathrm{r}=-0.679, \mathrm{P}<0.001)$.

In paediatric MPP patients, the miR-10a-3p expression level showed a positive correlation with WBC, ESR, and CRP (all $\mathrm{P}<0.05$ ). The COX11 mRNA expression level showed a positive correlation with ESR and CRP (both $\mathrm{P}<0.05)$ (Table 3).

Table 2 Clinical and laboratory indicators of paediatric MPP patients

\begin{tabular}{lc}
\hline Indicator & Result \\
\hline Cough $(\mathrm{n}, \%)$ & $93(95.9)$ \\
Fever $(\mathrm{n}, \%)$ & $70(72.2)$ \\
Pulmonary rales $(\mathrm{n}, \%)$ & $62(63.9)$ \\
Wheezing $(\mathrm{n}, \%)$ & $21(21.6)$ \\
Nasal congestion and runny nose $(\mathrm{n}, \%)$ & $20(20.6)$ \\
WBC $\left(\bar{x} \pm \mathrm{S}, \times 10^{9} / \mathrm{L}\right)$ & $8.93 \pm 2.46$ \\
Neut $\left(\bar{x} \pm \mathrm{S}, \times 10^{9} / \mathrm{L}\right)$ & $4.67 \pm 1.32$ \\
PLT $\left(\bar{x} \pm \mathrm{S}, \times 10^{9} / \mathrm{L}\right)$ & $289.63 \pm 21.35$ \\
ESR $(\bar{x} \pm \mathrm{S}, \mathrm{mm} / \mathrm{h})$ & $17.78 \pm 4.12$ \\
CRP $(\bar{x} \pm \mathrm{s}, \mathrm{mg} / \mathrm{L})$ & $9.65 \pm 2.52$
\end{tabular}

MPP, Mycoplasma pneumoniae pneumonia; WBC, white blood cell; Neut, neutrophil; PLT, platelet; ESR, erythrocyte sedimentation rate; CRP, C-reactive protein.

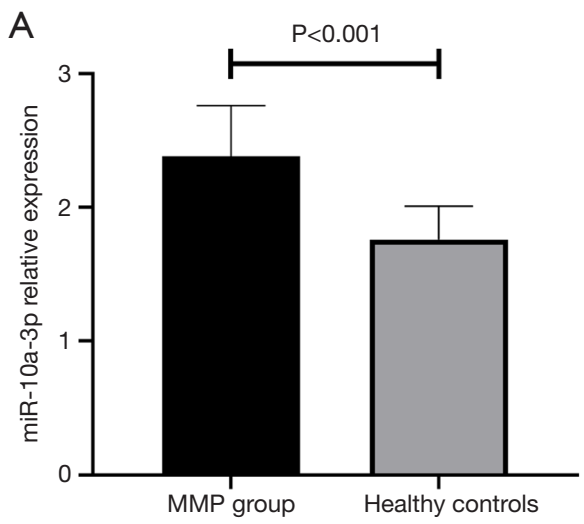

Validation of the targeting relationship between miR-10a$3 p$ and COX11 gene

As shown in Figure 2, miR-10a-3p and the COX11 mRNA had compatible binding sites. The dual luciferase assay confirmed that COX11 was a target mRNA of miR-10a-3p.

\section{The expression level of miR-10a-3p after stimulation of THP-1 cells by Mp-LAMPs}

After stimulation by the blank culture medium or MpLAMPs, the miR-10a-3p expression levels after 12, 24, and $48 \mathrm{~h}$ were detected. The results showed that the miR-10a$3 \mathrm{p}$ expression levels in THP-1 cells in the experimental group after 12, 24, and $48 \mathrm{~h}$ were all significantly higher than those in the control group $(\mathrm{P}<0.05)$. In addition, with longer stimulation time, the miR-10a-3p expression level gradually increased $(\mathrm{P}<0.05)$ (Figure 3$)$.

\section{Effect of transfecting the miR-10a-3p mimic and inbibitor in THP-1 cells}

After transfection, the relative expression level of miR$10 a-3 p$ in the mimic group was $3.53 \pm 0.57$, which was significantly higher than that in the mimic NC group $(2.12 \pm 0.35)$, inhibitor group $(1.32 \pm 0.23)$, or inhibitor NC group $(2.16 \pm 0.32)(\mathrm{P}<0.001)$. The relative expression level of $\mathrm{miR}-10 \mathrm{a}-3 \mathrm{p}$ in the inhibitor group was significantly lower than that in the other three groups $(\mathrm{P}<0.001)$ (Figure 4). These results indicate that the transfection was successful.

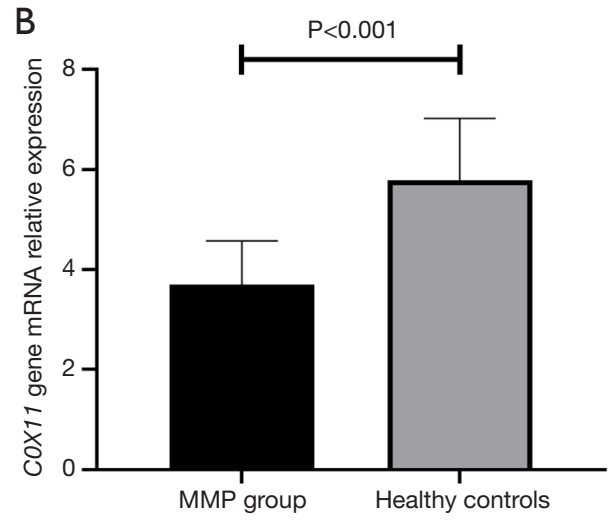

Figure 1 Relative expression levels of miR-10a-3p and COX11 mRNA in paediatric MPP patients and children in the healthy control group. (A) Relative expression of miR-10a-3p; (B) Relative expression of COX11 gene mRNA. MPP, Mycoplasma pneumoniae pneumonia. 
Table 3 Correlation between the expression levels of miR-10a-3p and COX11 mRNA and laboratory indicators of paediatric MPP patients

\begin{tabular}{lccccc}
\hline \multirow{2}{*}{ Indicator } & \multicolumn{3}{c}{ miR-10a-3p } & \multicolumn{2}{c}{ COX11 mRNA } \\
\cline { 2 - 5 } & $\mathrm{r}$ & $\mathrm{P}$ & $\mathrm{r}$ & 0.438 \\
WBC $\left(\times 10^{9} / \mathrm{L}\right)$ & 0.643 & 0.013 & 0.482 & 0.367 \\
Neut $\left(\times 10^{9} / \mathrm{L}\right)$ & 0.375 & 0.266 & 0.398 & 0.251 \\
PLT $\left(\times 10^{9} / \mathrm{L}\right)$ & 0.632 & 0.102 & 0.635 & 0.024 \\
ESR $(\mathrm{mm} / \mathrm{h})$ & 0.763 & $<0.001$ & 0.589 & 0.043 \\
CRP $(\mathrm{mg} / \mathrm{L})$ & 0.684 & 0.016 & & \\
\hline
\end{tabular}

MPP, Mycoplasma pneumoniae pneumonia; WBC, white blood cell; Neut, neutrophil; PLT, platelet; ESR, erythrocyte sedimentation rate; CRP, C-reactive protein.

A

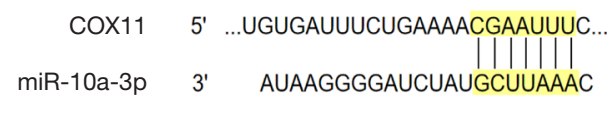

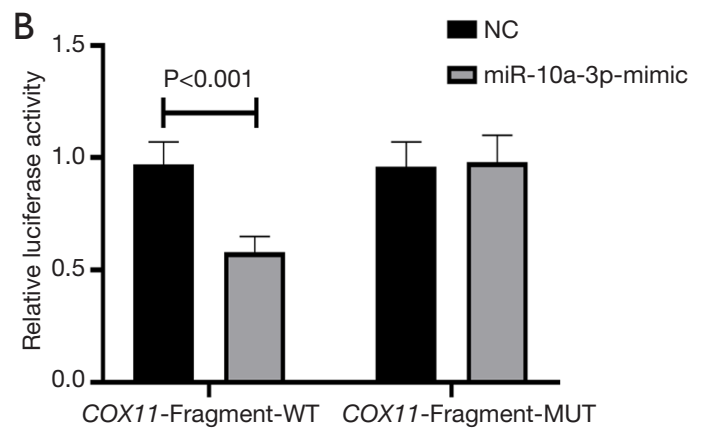

Figure 2 Validation of the targeting relationship between miR-10a-3p and COX11. (A) Binding sites between miR-10a-3p and COX11. (B) Dual luciferase reporter assay results.

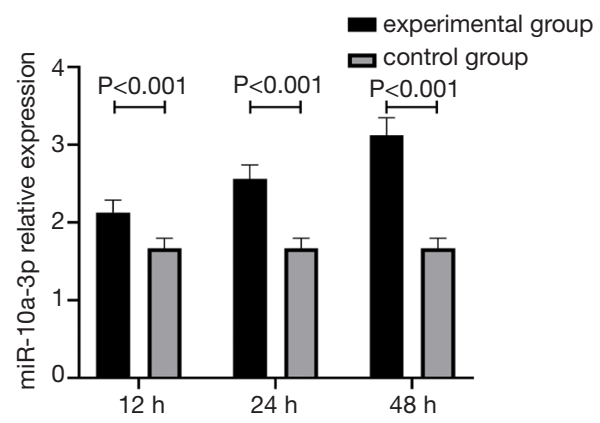

Figure 3 miR-10a-3p expression in THP-1 cells after MPP LAMP stimulation. MPP, Mycoplasma pneumoniae pneumonia; LAMP, lipid-associated membrane protein.

\section{Comparison of COX11 mRNA expression levels in THP-1 cells after transfection}

After transfection, the COX11 mRNA expression level in the mimic group was $2.82 \pm 0.41$, which was significantly lower than that in the mimic NC group $(4.14 \pm 0.65)$, inhibitor group $(6.03 \pm 0.95)$, or inhibitor NC group $(4.22 \pm 0.72)(\mathrm{P}<0.001)$. COX11 mRNA expression in the inhibitor group was significantly higher than that in the other three groups $(\mathrm{P}<0.001)$ (Figure 5).

\section{Changes in expression levels of NF- $\mathrm{\kappa B}$ signalling pathway- related proteins and CRP in THP-1 cells after transfection}

After transfection, I $\mathrm{I} B \alpha$ expression significantly decreased and $\mathrm{p}-\mathrm{IKK} \alpha / \beta, \mathrm{p}-\mathrm{p} 65$, and CRP significantly increased in the mimic group, whereas I $\mathrm{B} \alpha$ expression significantly increased and $\mathrm{p}-\mathrm{IKK} \alpha / \beta, \mathrm{p}-\mathrm{p} 65$, and CRP significantly decreased in the inhibitor group (Figure 6).

\section{Discussion}

miR-10a-3p expression was upregulated in MPP patients, COX11 expression decreased in MPP patients, and miR$10 \mathrm{a}-3 \mathrm{p}$ and COX11 both had significant correlations with clinical laboratory indicators of the MPP patients. In vitro 


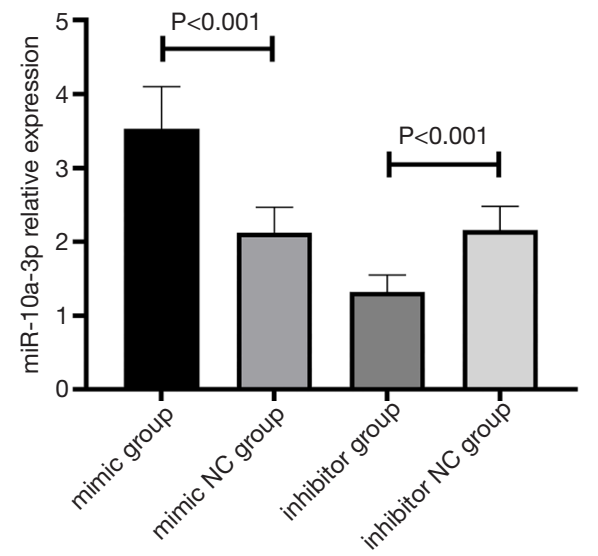

Figure 4 Relative expression levels of miR-10a-3p in all groups after transfection.

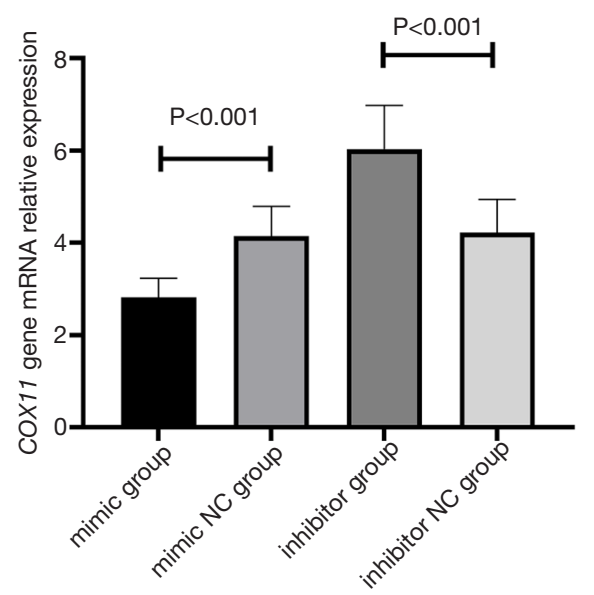

Figure 5 mRNA expression of COX11 gene in each group after transfection.

experiments confirmed the targeting relationship between miR-10a-3p and COX11. For the first time, this study reported that upregulation of miR-10a-3p in MPP could target COX11 to promote the development and progression of paediatric MPP. These results provide a reference for understanding the pathogenesis of MPP and developing targeted therapies for this disease.

miR-10a-3p plays an important role in the regulation of immune function (11). Our study showed that the relative expression level of miR-10a-3p in the MPP group was $2.38 \pm 0.52$, significantly higher than the $1.76 \pm 0.38$ in the control group. When we analysed the correlation between the miR-10a-3p expression level and laboratory inflammatory indicators, we found a positive correlation between miR-10a-3p expression level and WBC, ESR, and CRP. Indicators such as WBC, ESR, and CRP play a role in the evaluation of pneumonia and the determination of treatment efficacy and are associated with progression of pneumonia in a certain sense $(9,17)$. These results suggest that upregulation of miR-10a-3p expression in paediatric MPP might participate in disease development and progression. However, further functional experiments are required to validate this hypothesis.

By searching the TargetScan database, we found that COX11 was a downstream target mRNA of miR-10a$3 p$. Therefore, we investigated whether miR-10a-3p in paediatric MPP participated in disease development and progression by regulating COX11. First, the targeting relationship between miR-10a-3p and COX11 was confirmed. The dual luciferase reporter assay confirmed that COX11 was a target mRNA of miR-10a-3p. The relative expression level of COX11 mRNA in the paediatric MPP group was significantly lower than that in the healthy control group, and it showed a positive correlation with ESR and CRP. Therefore, COX11 expression also had a certain relationship with the progression of paediatric MPP. Next, we calculated the correlation between miR$10 a-3 p$ expression and the relative expression level of COX11 mRNA in paediatric MPP patients, finding a negative correlation. This result preliminarily suggested the correlation between increased miR-10a-3p expression and downregulated COX11 expression.

COX11 participates in important physiological processes in organisms, including metabolism, apoptosis, energy supply, and reactive oxygen production and plays an important role in cancer and respiratory diseases such as chronic obstructive pulmonary disease (COPD) $(18,19)$. Reduced COX activity can result in an increased apoptosis rate in pulmonary vascular endothelial cells of COPD patients to aggravate the disease and the clinical respiratory system symptoms of patients (20).

To further validate the role of miR-10a-3p in development and progression of paediatric MPP, in vitro cell experiments were performed. Under the stimulation of Mp-LAMPs, miR-10a-3p expression significantly increased in THP-1 cells, indicating that miR-10a-3p participated in the pathogenesis of MPP. miRNAs are a group of conserved RNAs. They have good stability and are not influenced by endogenous RNases. Therefore, miR-10a-3p has the potential to be used as a molecular marker for disease diagnosis after future studies confirm its role.

To validate the effect of miR-10a-3p on the COX11 


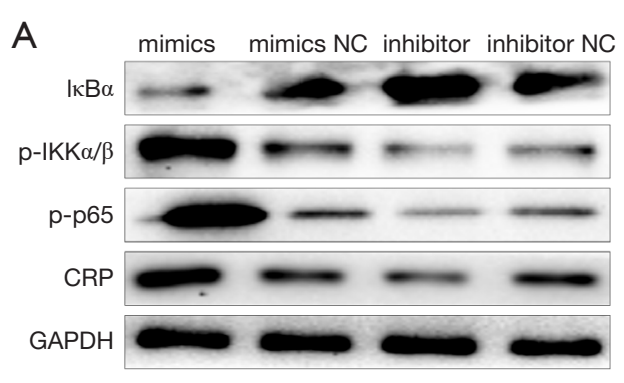

C
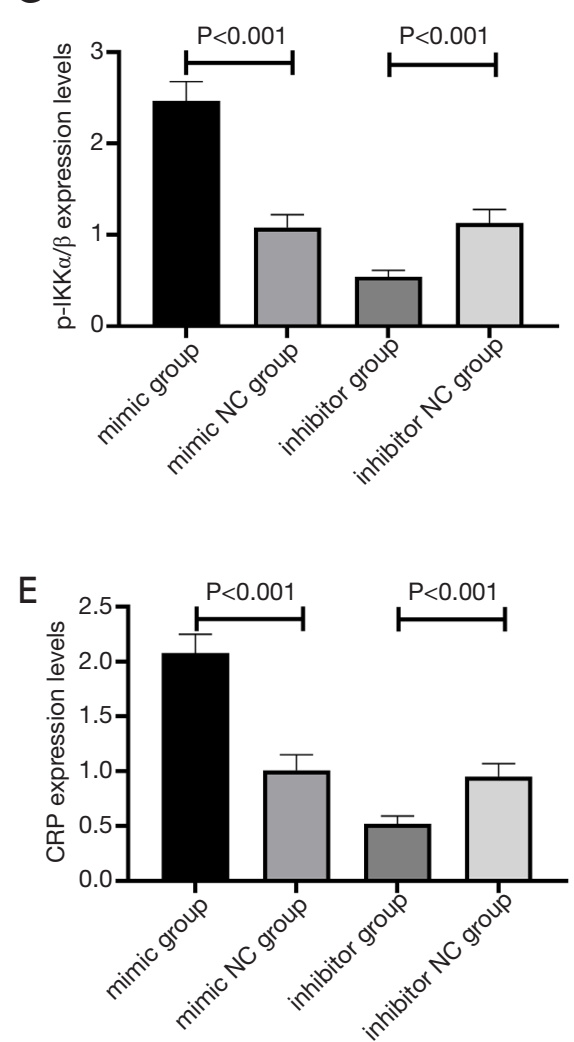

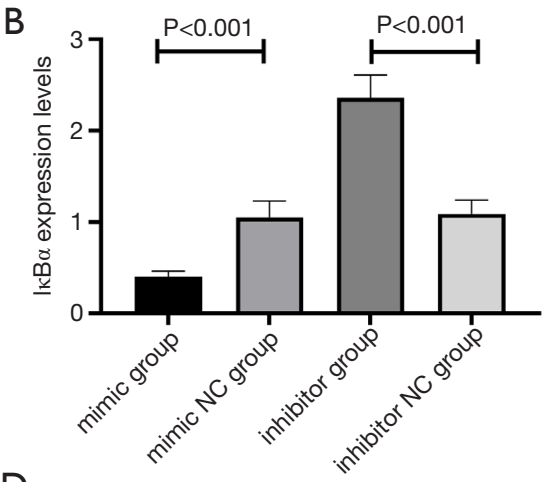

D

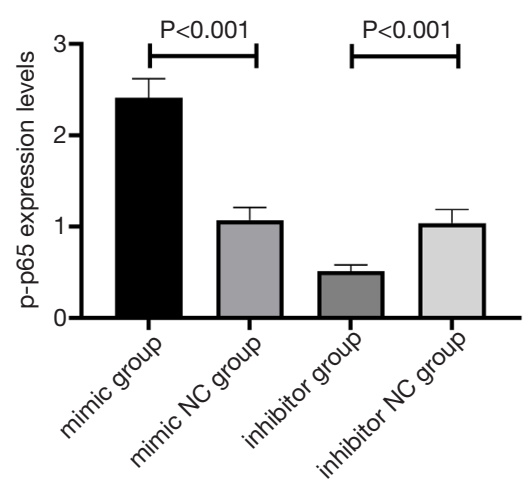

Figure 6 Expression levels of NF- $\mathrm{BB}$ signalling pathway-related proteins and CRP after transfection. (A) Western Blotting protein bands for

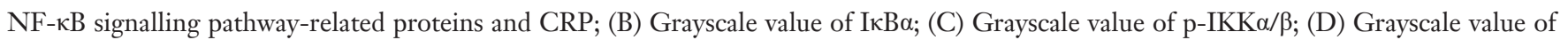
p-p65; (E) Grayscale value of CRP. NF- $\kappa$ B, nuclear factor- $\kappa \mathrm{B}$; CRP, C-reactive protein.

expression level, we treated THP-1 cells with an miR-10a$3 \mathrm{p}$ mimic and an inhibitor. The results showed that the miR-10a-3p expression level significantly increased in the mimic group and significantly decreased in the inhibitor group, whereas the COX11 mRNA expression level significantly decreased in the mimic group and significantly increased in the inhibitor group. These results suggested that increased miR-10a-3p expression might downregulate the COX11 expression level as part of its function.

Previous studies (21) show that activation of the NF$\kappa \mathrm{B}$ signalling pathway plays an important role in the pathogenesis of MPP. As a key anti-apoptotic transcription factor in immune cells such as neutrophils, NF- $\mathrm{BB}$ is very important in the regulation of immune responses and inflammatory processes. In the resting state, NF- $\kappa B$ exists as the p50/p65 dimer. In the cytoplasm, NF- $\mathrm{\kappa B}$ interacts 
with $\mathrm{I} \kappa \mathrm{B} \alpha$ to go into the inhibitory state. I $\kappa \mathrm{B} \alpha$ can be degraded by $\mathrm{p}-\mathrm{IKK} \alpha / \beta$ through the ubiquitination pathway, thus leaving NF- $\kappa \mathrm{B}$ in a free state. In addition, $\mathrm{p}-\mathrm{IKK} \alpha /$ $\beta$ can phosphorylate $\mathrm{p} 65$ to activate the NF- $\mathrm{kB}$ signalling pathway. Previous studies (22) had showed that many miRNAs were involved in NF- $\mathrm{\kappa B}$ signaling pathway and played an important role in the regulation of cell apoptosis. For example, miR-146a inhibited NF expression in THP1 cells- $\kappa \mathrm{B}$ signaling pathway and down regulated the expression of pro-inflammatory cytokines; Mir-17-5p can activate $\mathrm{NF}-\kappa \mathrm{B}$ signaling pathway promoted macrophage proliferation and inflammatory response (23). In this study, we also explored the effect of mir-10a-3p on NF$\kappa \mathrm{B}$ signaling pathway. Our results showed that after THP1 cells were treated with the miR-10a-3p mimic (inhibitor), IкB $\alpha$ expression significantly decreased (increased) and $\mathrm{p}-\mathrm{IKK} \alpha / \beta$ and $\mathrm{p}-\mathrm{p} 65$ protein expression significantly increased (decreased). In addition, CRP significantly increased in the mimic group and significantly decreased in the inhibitor group. CRP plays an important protective role in the innate immune process in the body. It can activate complements and enhance the phagocytic capacity of phagocytes to clear pathogenic microorganisms that infect the body, as well as damaged, necrotic, and apoptotic tissue cells after the body suffers from inflammatory stimulation such as microbial infection or tissue damage. In this study, we confirmed in vitro that the overexpression of miR-10a$3 p$ targeted COX11, and the increased expression of miR$10 \mathrm{a}-3 \mathrm{p}$ promoted the production of immune inflammatory factors by activating the NF- $\kappa \mathrm{B}$ signaling pathway, thus playing a role in the pathogenesis of the disease

In summary, in paediatric MPP, increased miR-10a$3 \mathrm{p}$ expression could downregulate the COX11 expression level, activate the NF- $\mathrm{KB}$ signalling pathway, and thereby participate in disease development and progression.

\section{Acknowledgments}

Funding: This work was supported by Qiqihar Science and Technology Bureau of Heilongiiang Province Joint Guidance Project [LHYD202037].

\section{Footnote}

Reporting Checklist: The authors have completed the TREND reporting checklist. Available at https://dx.doi. org/10.21037/jtd-21-710
Data Sharing Statement: Available at https://dx.doi. org/10.21037/jtd-21-710

Conflicts of Interest: All authors have completed the ICMJE uniform disclosure form (available at https://dx.doi. org/10.21037/jtd-21-710). All authors report funding from Qiqihar Science and Technology Bureau of Heilongjiang Province Joint Guidance Project [LHYD202037].

Ethical Statement: The authors are accountable for all aspects of the work in ensuring that questions related to the accuracy or integrity of any part of the work are appropriately investigated and resolved. The study was conducted in accordance with the Declaration of Helsinki (as revised in 2013). The Ethics Committee of the First Affiliated Hospital of Qiqihar Medical College in Heilongjiang Province approved the research protocol (LHYD202037). The need for written informed consent was waived because of the retrospective study design.

Open Access Statement: This is an Open Access article distributed in accordance with the Creative Commons Attribution-NonCommercial-NoDerivs 4.0 International License (CC BY-NC-ND 4.0), which permits the noncommercial replication and distribution of the article with the strict proviso that no changes or edits are made and the original work is properly cited (including links to both the formal publication through the relevant DOI and the license). See: https://creativecommons.org/licenses/by-nc-nd/4.0/.

\section{References}

1. He H, Wang X, Xiao Y, et al. Comparative efficacy and safety of traditional Chinese patent medicine in the treatment of Mycoplasma pneumoniae pneumonia in children: A protocol for systematic review and metaanalysis. Medicine (Baltimore) 2020;99:e23747 .

2. Yin L, Ma Y, Wang W, et al. The critical function of miR1323/Il6 axis in children with Mycoplasma pneumoniae pneumonia. J Pediatr (Rio J) 2021;97:552-8.

3. Mărginean CO, Meliţ LE, Simu I, et al. The Association Between Mycoplasma pneumoniae and Chlamydia pneumoniae, a Life-Threatening Condition in Small Children-A Case Report and a Review of the Literature. Front Pediatr 2020;8:558941.

4. Tsai TA, Tsai CK, Kuo KC, et al. Rational stepwise approach for Mycoplasma pneumoniae pneumonia in 
children. J Microbiol Immunol Infect 2021;54:557-65.

5. Lai JF, Zindl CL, Duffy LB, et al. Critical role of macrophages and their activation via MyD88-NFкB signaling in lung innate immunity to Mycoplasma pneumoniae. PLoS One 2010;5:e14417.

6. Zhao QY, Shi SJ, Sun DQ, et al. Correlation between galectin-3 level in bronchoalveolar lavage fluid and cellular immunity in children with refractory Mycoplasma pneumoniae pneumonia. Zhongguo Dang Dai Er Ke Za Zhi 2019;21:150-4.

7. Sun D. Clinical characteristics and expression of miRNA492 in peripheral blood of children with Mycoplasma pneumoniae pneumonia in Suzhou area. Suzhou University; 2017.

8. Chu C, Lei X, Li Y, et al. High expression of miR-222-3p in children with Mycoplasma pneumoniae pneumonia. Ital J Pediatr 2019;45:163.

9. Qi X, Sun X, Li X, et al. Significance changes in the levels of myocardial enzyme in the child patients with Mycoplasma Pneumoniae Pneumonia. Cell Mol Biol (Noisy-le-grand) 2020;66:41-5.

10. Weidle UH, Birzele F, Nopora A. microRNAs Promoting Growth of Gastric Cancer Xenografts and Correlation to Clinical Prognosis. Cancer Genomics Proteomics 2021;18:1-15.

11. Zhang X, Huang F, Yang D, et al. Identification of miRNA-mRNA Crosstalk in Respiratory Syncytial Virus- (RSV-) Associated Pediatric Pneumonia through Integrated miRNAome and Transcriptome Analysis. Mediators Inflamm 2020;2020:8919534.

12. Mirijello A, La Marca A, D'Errico MM, et al. Venous thromboembolism during mycoplasma pneumoniae infection: case report and review of the literature. Eur Rev Med Pharmacol Sci 2020;24:10061-8.

13. Pfausler B, Engelhardt K, Kampfl A, et al. Postinfectious central and peripheral nervous system diseases complicating Mycoplasma pneumoniae infection. Report of three cases and review of the literature. Eur J Neurol 2002;9:93-6.

Cite this article as: Li W, Ding X, Zhao R, Xiong D, Xie Z, $\mathrm{Xu}$ J, Tan M, Li C, Yang C. The role of targeted regulation of COX11 by miR-10a-3p in the development and progression of paediatric mycoplasma pneumoniae pneumonia. J Thorac Dis 2021;13(9):5409-5418. doi: 10.21037/jtd-21-710
14. You G, Cao H, Yan L, et al. MicroRNA-10a-3p mediates Th17/Treg cell balance and improves renal injury by inhibiting REG3A in lupus nephritis. Int Immunopharmacol 2020;88:106891.

15. Sen P, Ghosal S, Hazra R, et al. Transcriptomic analyses of gene expression by CRISPR knockout of miR-214 in cervical cancer cells. Genomics 2020;112:1490-9.

16. Zhang S, Liu H, Amarsingh GV, et al. Restoration of myocellular copper-trafficking proteins and mitochondrial copper enzymes repairs cardiac function in rats with diabetes-evoked heart failure. Metallomics 2020;12:259-72.

17. Huang X, Li D, Liu F, et al. Clinical significance of D-dimer levels in refractory Mycoplasma pneumoniae pneumonia. BMC Infect Dis 2021;21:14.

18. Radin I, Mansilla N, Rödel G, et al. The Arabidopsis COX11 Homolog is Essential for Cytochrome c Oxidase Activity. Front Plant Sci 2015;6:1091.

19. Sun M, Zuo X, Li R, et al. Vascular endothelial growth factor recovers suppressed cytochrome c oxidase activity by restoring copper availability in hypertrophic cardiomyocytes. Exp Biol Med (Maywood) 2014;239:1671-7.

20. Wiegman CH, Li F, Ryffel B, et al. Oxidative Stress in Ozone-Induced Chronic Lung Inflammation and Emphysema: A Facet of Chronic Obstructive Pulmonary Disease. Front Immunol 2020;11:1957.

21. Chen C, Wang J, Chen J, et al. Morusin alleviates mycoplasma pneumonia via the inhibition of Wnt/ $\beta$-catenin and NF- $\kappa$ B signaling. Biosci Rep 2019;39:BSR20190190.

22. Zhou C, Zhao L, Wang K, et al. MicroRNA-146a inhibits NF- $\kappa \mathrm{B}$ activation and pro-inflammatory cytokine production by regulating IRAK1 expression in THP-1 cells. Exp Ther Med 2019;18:3078-84.

23. An JH, Chen ZY, Ma QL, et al. LncRNA SNHG16 promoted proliferation and inflammatory response of macrophages through miR-17-5p/NF- $\mathrm{BB}$ signaling pathway in patients with atherosclerosis. Eur Rev Med Pharmacol Sci 2019;23:8665-77. 